\title{
MAXIMUM PRINCIPLES, GRADIENT ESTIMATES, AND WEAK SOLUTIONS FOR SECOND ORDER PARTIAL DIFFERENTIAL EQUATIONS
}

BY

\author{
WILLIAM BERTIGER
}

\begin{abstract}
Weak solutions to second order elliptic equations and the first derivatives of these solutions are shown to satisfy $L^{p}$ bounds. Classical second order equations with nonnegative characteristic form are also considered. It is proved that auxiliary functions of the gradient of a solution must satisfy a maximum principle. This result is extended to higher order derivatives and systems.
\end{abstract}

1. Introduction. We consider both weak and classical solutions of second order partial differential equations and solutions of second order systems. We obtain a priori bounds for the derivatives of the solutions; in addition, maximum principles are established for functions of the gradient and for higher order derivatives.

Let $x=\left(x_{1}, x_{2}, \ldots, x_{n}\right)$; by $U_{, i}(x)$ we denote the partial derivative of $U$ with respect to $x_{i}$. Similarly $U_{i j}=\partial^{2} U / \partial x_{i} \partial x_{j}$, and so on for higher derivatives. The results in $\$ 2$ concern weak derivatives, while the theorems in $\$ 3$ pertain to classical derivatives. The symbol $L^{p}(\Omega)$ will denote the usual Lebesgue space with norm $\|U\|_{p}=\left(\int_{\Omega}|U|^{p} d x\right)^{1 / p}$. Throughout $\$ 2$ we abbreviate $L^{p}(\Omega)$ by $L^{p}$, and all integrals are over a bounded domain $\Omega \subset \mathbf{R}^{n}$. The symbol $H^{m, p}(\Omega)$ (abbreviated $H^{m, p}$ ) denotes the Sobolev space with $m$ weak derivatives in $L^{p}$. The symbol $H_{0}^{m, p}(\Omega)$ (abbreviated $H_{0}^{m, p}$ ) denotes the completion of $C_{0}^{\infty}(\Omega)$ (infinitely differentiable functions with compact support in $\Omega$ ) in the norm of $H^{m p}$. The summation convention is used, i.e., repeated indices are summed.

In $\$ 2$ we consider elliptic equations for a function $U$ of the form

$$
\int_{\Omega}\left[a^{i j} U_{, i j} q+b^{i} U_{, i} q+C U q\right] d x=\int_{\Omega} f q d x
$$

for all $q \in H^{1,4}$.

The first theorem we establish is similar to a result proved by Miranda [2] for semicoupled systems. Miranda's theorem concerns $L^{\infty}$ bounds for $U$. We prove

Received by the editors August 10, 1976.

AMS (MOS) subject classifications (1970). Primary 35B45.

- American Mathematical Society 1978 
THEOREM 1. Let $U$ be an element of $H^{1, p} \cap L^{8 \alpha /(1-4 / p)} \cap H_{0}^{2,2}, p \geqslant 4$, $\alpha$ an integer $\geqslant 1$. Let $f$ belong to $L^{2 \alpha+2}$. Assume that $a^{i j}, b^{i}, C$ are in $H^{1,4}$ and that the matrix $\left(a^{i j}\right)$ is positive definite and symmetric; denote by $\left(A^{i j}\right)$ the inverse of $\left(a^{i j}\right)$. Assume there is a positive constant $C_{0}$ such that

$$
(2 \alpha+1)^{-1}\left[\frac{1}{2} A^{i j} b^{j} a_{, m}^{i m}-\frac{1}{4} A^{i j} a_{, m}^{i m} a_{, k}^{j k}-\frac{1}{4} A^{i j} b^{i} b^{j}\right]-C \geqslant C_{0}
$$

or, equivalently,

$$
-(2 \alpha+1)^{-1} \frac{1}{4} A^{i j}\left(a_{, k}^{i m}-b^{i}\right)\left(a_{, k}^{j k}-b^{j}\right)-C \geqslant C_{0} .
$$

If $U$ is a solution of (1.1) then

$$
\|U\|_{2 \alpha+2} \leqslant C_{0}^{-1}\|f\|_{2 \alpha+2} \text {. }
$$

Miranda considers equations in divergence form with $b^{i} \in L^{n}, C \in L^{n / 2}$. He assumes that the coefficients $a^{i j}$ are uniformly bounded and that the matrix $\left(a^{i j}\right)$ is uniformly positive definite. Stampacchia [3] proves under conditions similar to those of Miranda that weak solutions of uniformly elliptic equations satisfy a weak maximum principle (i.e. the solution is bounded by its boundary values). Theorem 1 may be used to prove the uniqueness of solutions to (1.1).

Theorem 1 is used in the proof of Theorem 2 which yields bounds for the derivatives of solutions of equation (1.1).

TheOREM 2. Suppose $a^{i j}, b^{i}, C$ are elements of $H^{1,4}, 1 \leqslant i, j \leqslant n, f$ is an element of $H_{0}^{1,2} \cap H^{1,4 \alpha+4}$ and $U$ is an element of $H^{2,2 p} \cap H^{1,16 \alpha /(1-4 / p)} \cap$ $H_{0}^{3,4}, p \geqslant 4, \alpha$ an integer $\geqslant 1$. Assume, as in Theorem 1, that the coefficients satisfy (1.2) and that

$\exists \gamma \geqslant 0$ such that

$$
\begin{aligned}
\int\left[-\frac{1}{4} A^{i j} a_{, l}^{k j} V_{, l} a_{, m}^{i k} V_{, m} q+2 \gamma a^{i j} V_{, i} V_{j} q-V_{, k} V_{, k} q\right. \\
\left.\quad-2 b_{, k}^{i} V_{, i} V_{, k} q-C V_{, k} V_{, k} q-\left(C_{, k} V_{, k}\right)^{2} q\right] d x \geqslant 0
\end{aligned}
$$

for all nonnegative $q$ in $H^{1,4}$, and for all $V$ in $H^{2,2 p} \cap H^{1,16 \alpha /(1-4 / p)} \cap H_{0}^{3,4}$. Let $W=U_{, k} U_{, k}+\gamma U^{2}$. If $U$ is a solution of (1.1) then

$$
\|W\|_{2 \alpha+2} \leqslant C_{0}^{-1}\left\|f_{, k} f_{, k}+(n+\gamma) U^{2}+\gamma U^{2}\right\|_{2 \alpha+2} \text {. }
$$

If, in addition, $U$ is an element of $L^{(16 \alpha+8) /(1-4 / p)}$ or if $C_{0}>1$, then

$$
\|W\|_{2 \alpha+2} \leqslant C_{0}^{-1}\left\|f_{, k} f_{, k}+\gamma f^{2}\right\|_{2 \alpha+2} \text {. }
$$

The use of auxiliary function $W$ will be discussed further below.

$\$ 3$ is concerned with classical solutions of equations of the form

$$
a^{i j}(x, t) U_{, i j}+b^{i}(x, t) U_{, i}+C(x, t) U+U_{, t}=0
$$

where $U_{, t}=\partial U / \partial t$. $\left(a^{i j}\right)$ is a positive semidefinite symmetric matrix (i.e. 
$a^{i j} \xi_{i} \xi_{j} \geqslant 0$ for all $(x, t)$ and all real vectors $\left.\xi=\left(\xi_{1}, \ldots, \xi_{n}\right)\right)$. This equation includes parabolic and ultraparabolic equations. Oleinik and Radkevic in their book [5] discuss operators $L[U]=a^{i j} U_{, i j}+b^{i} U_{, i}+C U$ where $\left(a^{i j}\right)$ is positive semidefinite. In the last chapter of their book they discuss maximum principles for equations of this type which are due to Bony [8] and Aleksandrov [9]. They generalize previously known maximum principles which can be found, for example, in Protter and Weinberger [6]. The main result of this section is obtained by making use of these maximum principles to obtain bounds for the gradient of solutions to (1.4). More specifically, we consider equation (1.4) on a domain $\Omega=D \times T$ where $D$ is a bounded domain in $\mathbf{R}^{n}$ and $T=\left\{t_{1}<t<t_{2}\right\}$ is an open interval. We employ the auxiliary function $W=\left(U_{, k} U_{, k}+\left(U_{, t}\right)^{2}+1\right) e^{N t}$ where $N$ is a positive constant to be chosen. We then prove

THEOREM 3. Suppose that:

(1) $\left(a^{i j}\right)$ is a positive semidefinite symmetric matrix and $\left(a^{i j}(x, t)\right)$ may be extended as a positive semidefinite matrix to $\mathbf{R}^{n} \times \mathbf{R}$ such that $a^{i j}(x, t)$ is in $C^{2}\left(\mathbf{R}^{n} \times \mathbf{R}\right)$, with bounded second derivatives.

(2) $b^{i}$ and $C$ are in $C^{2}(\Omega)$ and are bounded together with their first partial derivatives.

(3) $U \in C^{3}(\Omega)$ and $U$ is uniformly bounded in $\Omega$.

Then if $U$ satisfies equation (1.4) the constant $N$ may be chosen sufficiently large so that $L[W] \geqslant 0$.

Once this theorem has been proved we may use the maximum principles of Bony or Aleksandrov to assert a maximum principle for the auxilliary function $W$, which implies bounds for the gradient of the solution. The auxiliary function $W=\left(U_{, k} U_{, k}+\left(U_{, t}\right)^{2}+1\right) e^{N t}$ was introduced by Bernstein and may be found in Il'in, Kalashnikov and Oleinik [4]. They make use of these functions in obtaining a priori estimates for parabolic equations. Similar results for elliptic equations are obtained by Protter and Weinberger [7] by considering the auxiliary function $W=U_{, k} U_{, k}+\gamma U^{2}, \gamma$ a constant. They prove if $U$ satisfies an elliptic equation then $W$ satisfies an elliptic inequality and therefore a maximum principle. They also apply this technique to higher order derivatives and systems. In $\$ 3$ we extend the main theorem stated above to higher order derivatives by considering the auxiliary function $W=\Sigma_{|\alpha|<K}\left(U_{, \alpha} U_{, \alpha}+1\right) e^{N t}$ where $\alpha$ is a multi-index which includes differentiation in $t$. We also extend the theorem to include weakly coupled systems of the form

$$
L^{\mu}[U]=a^{i j} U_{, i j}^{\mu}+b^{i} U_{, i}^{\mu}+U_{, t}^{\mu}+C^{\mu \nu} U^{\nu}=0, \quad \mu=1,2, \ldots, m
$$

( $\nu$ is summed from 1 to $m$ ). In particular, we consider $W=\left(U_{, k}^{\mu} U_{, k}^{\mu}+U_{, t}^{\mu} U_{, t}^{\mu}\right.$ $+1) e^{N t}$ and prove under conditions almost identical to the theorem stated 
above that $L[W]=a^{i j} W_{, i j}+b^{i} W_{, i}+W_{, t} \geqslant 0$.

This paper was written as a thesis under the supervision of Murray Protter. I would like to thank him for his invaluable advice and encouragement.

2. Weak solutions to second order elliptic equations. We first state two simple lemmas concerning integration by parts which will be used in the proofs of both theorems of this section.

Lemma A. Let $g \in H_{0}^{1,2}, f \in H^{1,2}$. Then $\int f g_{, i} d x=-\int f_{, i} g d x$.

LeMmA B. If $f$ and $g$ are elements of $H^{1,4}$ then $f g$ is an element of $H^{1,2}$ and $(f g)_{, i}=f_{, i} g+f g_{, i}$.

The proofs of these lemmas follow directly from the definitions of the spaces involved. One more lemma is necessary.

Lemma C. Suppose $U \in H^{1, p} \cap L^{4(\beta-1) /(1-4 / p)}, p \geqslant 4, \beta$ a positive integer (if $p=4$ and $\beta \geqslant 1$, then $\left.U \in H^{1,4} \cap L^{\infty}\right)$. Then $U^{\beta} \in H^{1,4}$ and $\left(U^{\beta}\right)_{, i}=$ $\beta U^{\beta-1} U_{, i}$.

Proof. The proof will be by induction on $\beta$. If $\beta=1, U \in H^{1, p}(\Omega)$ for some $p \geqslant 4$. Since $\Omega$ is bounded, $U \in H^{1,4}$ and there is nothing to prove. Assume the lemma is true for $\beta=k$. Suppose $U \in H^{1, p} \cap L^{4 k /(1-4 / p)}$. This implies $U \in H^{1, p} \cap L^{4(k-1) /(1-4 / p)}$. Therefore $U^{k} \in H^{1,4}$ and $\left(U^{k}\right)_{i}=$ $k U^{k-1} U_{, i}$. We wish to show that $U^{k+1} \in H^{1,4}$ and that $\left(U^{k+1}\right)_{, i}=(k+$ 1) $U^{k} U_{, i}$. First note that from Lemma $B, U^{k+1}$ has a weak derivative

$$
\left(U^{k+1}\right)_{, i}=\left(U^{k}\right)_{, i} U+U^{k} U_{, i}=k \cdot U^{k-1} U_{, i} U+U^{k} U_{, i}=(k+1) U^{k} U_{, i} \cdot
$$

We need now only show that $U^{k+1}$ and $\left(U^{k+1}\right)_{, i}$ are in $L^{4}$.

$$
\left\|U^{k+1}\right\|_{4} \leqslant\|U\|_{p}\left\|U^{k}\right\|_{4 /(1-4 / p)}, \quad\left\|U^{k} U_{, i}\right\|_{4} \leqslant\left\|U_{, i}\right\|_{p}\left\|U^{k}\right\|_{4 /(1-4 / p)} .
$$

The terms on the right side are bounded, by the assumptions on $U$.

We are considering elliptic equations for a function $U$ of the form

$$
\int_{\Omega}\left[a^{i j} U_{, i j} q+b^{i} U_{, i} q+C U q\right] d x=\int_{\Omega} f_{q} d x
$$

for all $q \in H^{1,4}$. Our first theorem concerns the boundedness of solutions to (2.1).

THEOREM 1. Let $U$ be an element of $H^{1, p} \cap L^{8 \alpha /(1-4 / p)} \cap H_{0}^{2,2}, p \geqslant 4, \alpha$ an integer $\geqslant 1$. Let $f$ belong to $L^{2 \alpha+2}$. Assume that $a^{i j}, b^{i}, C$ are in $H^{1,4}$ and that the matrix $\left(a^{i j}\right)$ is positive definite and symmetric; denote by $\left(A^{i j}\right)$ the inverse of $\left(a^{i j}\right)$. Assume there is a positive constant $C_{0}$ such that

$$
(2 \alpha+1)^{-1}\left[\frac{1}{2} A^{i j} b^{j} a_{, m}^{i m}-\frac{1}{4} A^{i j} a_{, m}^{i m} a_{, k}^{j k}-\frac{1}{4} A^{i j} b^{i} b^{j}\right]-C \geqslant C_{0}
$$

or, equivalently, 


$$
-(2 \alpha+1)^{-1} \frac{1}{4} A^{i j}\left(a_{, m}^{i m}-b^{i}\right)\left(a_{, k}^{j k}-b^{j}\right){ }_{1} C \geqslant C_{0} .
$$

If $U$ is a solution to (2.1) then

$$
\|U\|_{2 \alpha+2} \leqslant C_{0}^{-1}\|f\|_{2 \alpha+2} .
$$

Proof. With the above assumptions on $f, U$, and $q$ and the coefficients, note that the integrals

$$
\int\left[a^{i j} U_{, i j} q+b^{i} U_{, i} q+C U q\right] d x=\int f q d x
$$

exist and Lemmas A and B imply that

$$
\int\left[a^{i j} U_{, i} q_{j}+a_{j}^{i j} U_{, i} q-b^{i} U_{, i} q-C U q\right] d x=-\int f q d x .
$$

Let $q=U^{2 \alpha+1}$. By Lemma C, $q \in H^{1,4}$ (which is the reason for assuming $\left.U \in H^{1, p} \cap L^{8 \alpha /(1-4 / p)}\right)$. Also by Lemma $C$ we see that the weak derivative of $q$, denoted $q_{j}$, is $(2 \alpha+1) U_{j} U^{2 \alpha}$. Substituting this value of $q$ into the above integral equation (2.3), we obtain

$$
\begin{aligned}
& \int\left[a^{i j}(2 \alpha+1) U^{2 \alpha} U_{, i} U_{j}+a_{j}^{i j} U_{, i} U^{2 \alpha} U\right. \\
& \left.-b^{i} U_{, i} U^{2 \alpha} U-C U^{2 \alpha} U^{2}\right] d x=\int(-f) U^{2 \alpha} U .
\end{aligned}
$$

We now estimate the quadratic form in the integrand by completing the square.

$$
\begin{aligned}
0 \leqslant & (2 \alpha+1) a^{i j}\left[U_{, i}+\frac{1}{2}(2 \alpha+1)^{-1} A^{i m}\left(a_{, k}^{m k} U-b^{m} U\right)\right] \\
& \times\left[U_{j}+\frac{1}{2}(2 \alpha+1)^{-1} A^{r j}\left(a_{, l}^{r l} U-b^{r} U\right)\right] \\
= & (2 \alpha+1) a^{i j} U_{, i} U_{j}+a_{j}^{i j} U U_{, i}-b^{i} U U_{, i} \\
& +\frac{1}{4}(2 \alpha+1)^{-1} a^{i j} A^{i m} A^{r j} a_{, l}^{r l} a_{, k}^{m k} U^{2} \\
& +\frac{1}{4}(2 \alpha+1)^{-1} a^{i j} A^{i m} A^{r j} b^{r} b^{m} U^{2}-\frac{1}{4}(2 \alpha+1)^{-1} a^{i j} A^{i m} A^{r j} b^{r} a_{, k}^{m k} U^{2} \\
& -\frac{1}{4}(2 \alpha+1)^{-1} a^{i j} A^{i m} A^{r j} b^{m} a_{, l}^{r l} U^{2} \\
= & (2 \alpha+1) a^{i j} U_{, i} U_{j}+a_{j}^{i j} U U_{, i}-b^{i} U U_{, i} \\
& +\frac{1}{4}(2 \alpha+1)^{-1} A^{i j} a_{, m}^{i m} a_{, k}^{j k} U^{2}+\frac{1}{4}(2 \alpha+1)^{-1} A^{i j} b^{i} b^{j} U^{2} \\
& -\frac{1}{2}(2 \alpha+1)^{-1} A^{i j} b^{j} a_{, m}^{i m} U^{2} .
\end{aligned}
$$

From this computation we obtain the inequality

$$
\begin{gathered}
(2 \alpha+1)^{-1}\left[\frac{1}{2} A^{i j} b^{j} a_{, m}^{i m}-\frac{1}{4} A^{i j} a_{, m}^{i m} a_{, k}^{j k}-\frac{1}{4} A^{i j} b^{i} b^{j}\right] U^{2} \\
\leqslant(2 \alpha+1) a^{i j} U_{, i} U_{j}+a_{, j}^{i j} U U_{, i}-b^{i} U U_{, i} .
\end{gathered}
$$


Now using this inequality, the assumptions on the coefficients, (2.2) and (2.4) we conclude

$$
\int C_{0}|U|^{2 \alpha+2} d x \leqslant \int(-f)|U|^{2 \alpha}|U| d x
$$

An application of Hölder's inequality gives

$$
\begin{gathered}
\int C_{o}|U|^{2 \alpha+2} d x \leqslant\|f\|_{2 \alpha+2}\left[\int\left(|U|^{2 \alpha+2}\right) d x\right]^{(2 \alpha+1) /(2 \alpha+2)}, \\
C_{0}\|U\|_{2 \alpha+2}^{2 \alpha+2} \leqslant\|f\|_{2 \alpha+2}\|U\|_{2 \alpha+2}^{2 \alpha+1}, \quad\|U\|_{2 \alpha+2} \leqslant C_{0}^{-1}\|f\|_{2 \alpha+2} .
\end{gathered}
$$

REMARK. If $U \in H^{1,4} \cap L^{\infty} \cap H_{0}^{2,2}$ and (2.2) holds for all $\alpha$ sufficiently large, then by letting $\alpha$ tend to infinity we get

$$
\|U\|_{\infty} \leqslant C_{0}^{-1}\|f\|_{\infty}
$$

In Theorem 2 we obtain bounds on the weak derivatives of solutions to (2.1).

THEOREM 2. Suppose that $a^{i j}, b^{i}, C$ are elements of $H^{1,4}, 1 \leqslant i, j \leqslant n$, that $f$ is an element of $H_{0}^{1,2} \cap H^{1,4 \alpha+4}$, and that $U$ is an element of $H^{2,2 p} \cap$ $H^{1,16 \alpha /(1-4 / p)} \cap H^{3,4}, p \geqslant 4, \alpha$ an integer $\geqslant 1$. Assume as in Theorem 1 that the coefficients satisfy (2.2), that

$\exists \gamma \geqslant 0$ such that

$$
\begin{aligned}
\int[ & -\frac{1}{4} A^{i j} a_{, l}^{k j} V_{, l} a_{, m}^{i k} V_{, m} q+2 \gamma a^{i j} V_{, i} V_{, j} q \\
& \left.-V_{, k} V_{, k} q-2 b_{, k}^{i} V_{, i} V_{, k} q-C V_{, k} V_{, k} q-\left(C_{, k} V_{, k}\right)^{2} q\right] d x \geqslant 0
\end{aligned}
$$

for all nonnegative $q$ in $H^{1,4}$, and for any $V$ in $H^{2,2 p} \cap H^{1,16 \alpha /(1-4 / p)} \cap H_{0}^{3,4}$.

Let $W=U_{, k} U_{, k}+\gamma U^{2}$. If $U$ is a solution of (2.1) then

$$
\|W\|_{2 \alpha+2} \leqslant C_{0}^{-1}\left\|f_{, k} f_{, k}+(n+\gamma) U^{2}+\gamma U^{2}\right\|_{2 \alpha+2} .
$$

If, in addition, $U$ is an element of $L^{(16 \alpha+8) /(1-4 / p)}$ or if $C_{0}>1$, then

$$
\|W\|_{2 \alpha+2} \leqslant C_{0}^{-1}\left\|f_{, k} f_{, k}+\gamma f^{2}\right\|_{2 \alpha+2} \text {. }
$$

Proof. The proof consists of showing that $W$ satisfies an appropriate integral inequality. From this inequality we proceed as in the proof of Theorem 1 to obtain the desired result.

First we show that $W \in H^{1, p} \cap L^{8 \alpha /(1-4 / p)} \cap H_{0}^{2,2}$ (notice that this matches the hypothesis on $U$ in Theorem 1).

Since $W=U_{, k} U_{, k}+\gamma U^{2}$, we may use Lemma $C$ to obtain

$$
W_{, i}=2 U_{, k i} U_{k}+2 \gamma U U_{, i}
$$

and

$$
W_{, i j}=2 U_{, i j k} U_{, k}+2 U_{, i k} U_{j k}+2 \gamma U_{j} U_{, i}+2 \gamma U U_{, i j} .
$$


Hölder's Inequality gives $W \in H^{1, p} \cap L^{8 \alpha /(1-4 / p)}$. To see that $W \in H_{0}^{2,2}$, let $\left\{\phi^{m}\right\}$ be a sequence of functions such that $\phi^{m} \in C_{0}^{\infty}(\Omega)$ and $\phi^{m} \rightarrow U$ in $H^{3.4}$ as $m \rightarrow \infty$. We have $\phi_{, k}^{m} \phi_{, k}^{m}+\gamma\left(\phi^{m}\right)^{2} \in C_{0}^{\infty}(\Omega)$ (no summation on $m$ ). We show that $\phi_{, k}^{m} \phi_{, k}^{m}+\gamma\left(\phi^{m}\right)^{2} \rightarrow W$ in the norm of $H^{2,2}$. Then $W \in H_{0}^{2,2}$. We observe that

$$
\begin{aligned}
\left\|\phi_{, k}^{m} \phi_{, k}^{m}-U_{, k} U_{, k}\right\|_{2} & \leqslant\left\|\phi_{, k}^{m} \phi_{, k}^{m}-U_{, k} \phi_{, k}^{m}\right\|_{2}+\left\|\phi_{, k}^{m} U_{, k}-U_{, k} U_{, k}\right\|_{2} \\
& \leqslant\left\|\phi_{, k}^{m}\right\|_{4}\left\|\phi_{, k}^{m}-U_{, k}\right\|_{4}+\left\|U_{, k}\right\|_{4}\left\|\phi_{, k}^{m}-U_{, k}\right\|_{4} .
\end{aligned}
$$

Similarly all other appropriate terms converge.

Using the values of the weak derivatives of $W$ from above we have the following equation for $W$ :

$$
\begin{aligned}
\int\left[a^{i j} W_{, i j} q+b^{i} W_{, i} q+C W q\right] d x \\
=\int\left[2 a^{i j} U_{, i j k} U_{, k} q+2 a^{i j} U_{, i k} U_{j k} q\right. \\
+2 \gamma a^{i j} U_{j} U_{, i} q+2 \gamma a^{i j} U U_{, i j} q+2 b^{i} U_{, i k} U_{, k} q \\
\left.+2 \gamma b^{i} U U_{, i} q+C U_{, k} U_{, k} q+\gamma C U^{2} q\right] d x .
\end{aligned}
$$

Using the hypothesis on $U$ we eliminate the terms involving the weak dervatives of $U$ on the right side to obtain the inequality we seek for $W$.

If we restrict $q$ to $H^{2,4}$ then we may substitute $q_{, k}$ for $q$ in equation (2.1) and obtain

$$
\int\left[a^{i j} U_{, i j} q_{, k}+b^{i} U_{, i} q_{, k}+C U q_{, k}\right] d x=\int f q_{, k} d x
$$

Using Lemmas B and A we may integrate by parts to obtain

$$
\begin{aligned}
& \int\left[a_{, k}^{i j} U_{, i j} q+a^{i j} U_{, i j k} q+b_{, k}^{i} U_{, i} q+b^{i} U_{, i k} q\right. \\
&\left.+C_{, k} U q+C U_{, k} q\right] d x=\int f_{, k} q d x .
\end{aligned}
$$

If we restrict $q$ further to $H^{2,8}$ we may substitute $U q$ for $q$, or $q U_{, k}$ for $q$ and obtain, from (2.1),

$$
\int\left[2 \gamma a^{i j} U_{i j} U q+2 \gamma b^{i} U_{, i} U q+2 \gamma C U^{2} q\right] d x=\int 2 \gamma f U q d x
$$

and, from (2.6),

$$
\begin{aligned}
& \int\left[a_{, k}^{i j} U_{, i j} U_{, k} q+a^{i j} U_{, i j k} U_{, k} q+b_{, k}^{i} U_{, i} U_{, k} q\right. \\
& \left.\quad+b^{i} U_{, i k} U_{, k} q+C_{, k} U U_{, k} q+C U_{, k} U_{, k} q\right] d x=\int f_{, k} U_{, k} q d x
\end{aligned}
$$


Equations (2.7) and (2.8) hold for all $q \in H^{2,8}$. Using (2.7) and (2.8) we get

$$
\begin{aligned}
& \int\left[a^{i j} W_{, i j} q+b^{i} W_{, i} q+C W q\right] d x \\
& =\int\left[2 a^{i j} U_{, i k} U_{j k} q+2 \gamma a^{i j} U_{, i} U_{j} q\right. \\
& +2 \gamma f U q+2 f_{, k} U_{, k} q-2 a_{, k}^{i j} U_{, i j} U_{, k} q \\
& \left.\quad-2 b_{, k}^{i} U_{, i} U_{, k} q-2 C_{, k} U U_{, k} q-\gamma C U^{2} q-C U_{, k} U_{, k} q\right] d x
\end{aligned}
$$

for all $q \in H^{2,8}$; but it is also clear that (2.9) holds for all $q \in H^{1,4}$ by taking a sequence $\left\{\phi^{m}\right\}, \phi^{m} \in C^{\infty}$ and $\phi^{m} \rightarrow q$ in $H^{1,4}$. From this point on we assume that $q \geqslant 0$. Since $\left(a^{i j}\right)$ is positive definite we have

$$
\begin{aligned}
0 & \leqslant 2 a^{i j}\left(U_{, i k}-\frac{1}{2} A^{i m} a_{, l}^{k m} U_{, l}\right)\left(U_{j k}-\frac{1}{2} A^{j s} a_{, r}^{s k} U_{, r}\right) \\
& =2 a^{i j} U_{, i k} U_{, j k}-2 a_{, k}^{i j} U_{, i j} U_{, k}+\frac{1}{4} A^{i j} a_{, l}^{k j} U_{, l} a_{, m}^{i k} U_{, m} .
\end{aligned}
$$

Therefore

$$
-\frac{1}{4} A^{i j} a_{, l}^{k j} U_{, l} a_{, m}^{i k} U_{, m} \leqslant 2 a^{i j} U_{, i k} U_{j k}-2 a_{, k}^{i j} U_{, i j} U_{, k} .
$$

From (2.9)

$$
\begin{aligned}
& \int\left[a^{i j} W_{, i j} q+b^{i} W_{, i} q+C W q\right] d x \\
& \geqslant \int\left[-\frac{1}{4} A^{i j} a_{, l}^{k j} U_{, l} a_{, k}^{i k} U_{, m}+2 \gamma f U-\gamma C U^{2}\right. \\
& \left.\quad+2 \gamma a^{i j} U_{, i} U_{j}+2 f_{, k} U_{, k}-2 b_{, k}^{i} U_{, i} U_{, k}-2 C_{, k} U U_{, k}-C U_{, k} U_{, k}\right] q d x \\
& \geqslant \int\left[-\frac{1}{4} A^{i j} a_{, l}^{k j} U_{, l} a_{, k}^{i k} U_{, m}+2 \gamma a^{i j} U_{, i} U_{j}\right. \\
& \quad-C U_{, k} U_{, k}-f_{, k} f_{, k}-U_{, k} U_{, k} \\
& \left.\quad-2 b_{, k}^{i} U_{, i} U_{, k}-\left(C_{, k} U_{, k}\right)^{2}-n U^{2}-\gamma U^{2}-\gamma C U^{2}-\gamma f^{2}\right] q d x .
\end{aligned}
$$

From hypothesis (2.2) we note that $-C \geqslant C_{0}>0$. Using this and hypothesis (2.5) we obtain

$$
\begin{aligned}
\int\left[a^{i j} W_{, i j} q\right. & \left.+b^{i} W_{, i} q+C W q\right] \\
& \geqslant \int-\left[f_{, k} f_{, k}+(n+\gamma) U^{2}+\gamma f^{2}\right] q d x .
\end{aligned}
$$

If $C_{0}>1$ then we may use hypothesis (2.5) and the fact that we may choose $\gamma$ large enough such that $-n U^{2}-\gamma U^{2}-\gamma C U^{2} \geqslant 0$ to obtain 


$$
\int\left[a^{i j} W_{, i j} q+b^{2} W_{, i} q+C W q\right] d x \geqslant \int-\left[f_{, k} f_{, k}+\gamma f^{2}\right] q d x
$$

The assumptions on $U$ were seen in the beginning of the proof to imply that $W$ satisfies the conditions that $U$ does in Theorem 1. Furthermore ${ }^{-}-\left[f_{, k} f_{, k}+\right.$ $\left.(n+\gamma) U^{2}+\gamma f^{2}\right]$ and $-\left[f_{, k} f_{, k}+\gamma f^{2}\right]$ satisfy the same condition as $f$ does in Theorem 1. This is clear from the assumptions and Hölder's inequality. The proof of Theorem 1 can now be followed exactly (inequalities (2.10) and (2.11) above, instead of the equality of Theorem 1, cause no problems) to obtain

$$
\|W\|_{2 \alpha+2} \leqslant C_{0}^{-1}\left\|f_{, k} f_{, k}+(n+\gamma) U^{2}+\gamma f^{2}\right\|_{2 \alpha+2},
$$

and if $C_{0}>1$,

$$
\|W\|_{2 \alpha+2} \leqslant C_{0}^{-1}\left\|f_{, k} f_{, k}+\gamma f^{2}\right\|_{2 \alpha+2} .
$$

From Theorem 1 we know that if $U$ is also in $L^{(16 \alpha+8) /(1-4 / p)}$ then $\left\|U^{2}\right\|_{2 \alpha+2}$ $\leqslant C_{0}^{-1}\left\|f^{2}\right\|_{2 \alpha+2}$. We may use this to eliminate the term $(n+\gamma) U^{2}$ above. This completes the proof of Theorem 2.

Remarks. Condition (2.5) in Theorem 2 is always satisfied if the coefficients and their partial derivatives are uniformly bounded and $\left(a^{i j}\right)$ is uniformly positive definite. When $\left(a^{i j}\right)$ is uniformly positive definite there exists a constant $\mu$ such that $2 \gamma a^{i j} V_{, i} V_{j} \leqslant 2 \gamma \mu V_{, i} V_{, i}$. The other terms in condition (2.5) may be bounded by (constant) $V_{, i} V_{, i}$. Thus by choosing $\gamma$ large enough condition (2.5) is satisfied.

The coefficients will be continuous if $n<4$. This follows from a standard theorem on Sobolev spaces.

3. Equations with nonnegative characteristic form. Before stating the theorems of this section, it is convenient to state some maximum principles which will be useful. These may be found in the book by Oleinik and Radkevič [5] and are extensions by the translator of theorems due to Bony [8].

To state maximum principles for solutions of equations with nonnegative characteristic form (positive semidefinite principal part) we first make some definitions. Let $\bar{y}_{i}=\left(\alpha_{i 1}(x), \ldots, \alpha_{i n}(x)\right), i=1,2, \ldots, k$, denote a set of vectors defined on a domain $\Omega$ in $\mathbf{R}^{n}$ where $\alpha_{i j}(x) \in C^{\infty}(\Omega)$.

Definition. If $U$ is in $C^{\infty}(\Omega)$ then define $\bar{y}_{i} U=\alpha_{i j} U_{j}$. Define a bracket operation by $\left[\bar{y}_{i}, \bar{y}_{j}\right] U=\bar{y}_{j}\left(\bar{y}_{i}(U)\right)-\bar{y}_{i}\left(\bar{y}_{j}(U)\right)$. Define $\bar{E}\left(\bar{y}_{1}, \ldots, \bar{y}_{k}\right)$ to be the Lie algebra generated by $\bar{y}_{1}, \ldots, \bar{y}_{k}$; that is, $\varrho$ is the smallest $C^{\infty}$ module closed under the bracket operation and containing $\bar{y}_{1}, \bar{y}_{2}, \ldots, \bar{y}_{k}$. Define the rank of $\left\{\bar{y}_{1}, \ldots, \bar{y}_{k}\right\}$ at a point $x$ to be the dimension of the vector space formed by all $\bar{z}(x)$ such that $\bar{z} \in \mathcal{L}\left(\bar{y}_{1}, \ldots, \bar{y}_{k}\right)$. Define a trajectory of a vector field $\bar{y}_{i}$ to be a curve in $\Omega$ satisfying $d x_{j}(t) / d t=\alpha_{i j}(x)$.

TheOREM 3. Let $L[U]=a^{i j} U_{, i j}+b^{i} U_{, i}+C U \geqslant 0$ in a bounded domain 
$\Omega \subset \mathbf{R}^{n}$. Assume $\left(a^{i j}\right)$ is a positive semidefinite symmetric matrix; $a^{i j} \in$ $C^{\infty}(\Omega) ; C(x)<0$; and $a^{i j}, b^{i}, C$ are bounded. Let $\overline{L_{j}}=\left(a^{1 j}, \ldots, a^{n j}\right)$. If $U$ has a nonnegative maximum at a point $x\left(t_{0}\right)$ then $U$ is constant for the entire trajectory $x(t)$ of $\bar{L}_{j}, j=1, \ldots, n$, passing through $x\left(t_{0}\right)$.

THEOREM 4. Let $L[U] \geqslant 0$ as above with the same notation and conditions as in Theorem 3. Also assume that the rank of $\left\{\bar{L}_{1}, \ldots, \bar{L}_{n}\right\}$ is equal to $m \leqslant n$. Let $\bar{Q}=\left(b^{1}-a_{j}^{1 j}, b^{2}-a_{, j}^{2 j}, \ldots, b^{n}-a_{j}^{n j}\right)$. If $U(x)$ assumes a nonnegative maximum $M$ at a point $x_{0} \in \Omega$ then $U(x)=M$ at each point $x$ of $\Omega$ which may be joined with $x_{0}$ by a line consisting of a finite number of arcs of trajectories of the vector fields $\bar{L}_{j}(x)(j=1, \ldots, n)$ and $\bar{Q}(x)$ with the stipulation that when such a line is followed away from the point $x_{0}$, any arcs of trajectories of the field $\bar{Q}(x)$ are followed in the direction of the vector $\bar{Q}(x)$.

The proofs of the above two theorems may be found in $\S 1$ of Chapter III in Oleinik and Radkevič [5]. They note that the assumption $a^{i j} \in C^{\infty}(\Omega)$ is only for simplicity. We shall also need a lemma on positive semidefinite matrices which is stated and proved on p. 65 of [5].

Lemma. Let $a^{i j}(x) \xi_{i} \xi_{j} \geqslant 0$ for all $x$ in $\mathbf{R}^{m}$ and all $\xi=\left(\xi_{1}, \xi_{2}, \ldots, \xi_{m}\right) \in \mathbf{R}^{m}$, and suppose $a^{i j}(x) \in C^{2}\left(\mathbf{R}^{m}\right)$, and that the second order derivatives of $a^{i j}$ are uniformly bounded. Then for any $U \in C^{2}\left(\mathbf{R}^{m}\right)$,

$$
\left(a_{, k}^{i j} U_{, i j}\right)^{2} \leqslant M a^{i j} U_{, l i} U_{l j}
$$

where $M$ depends only on the second derivatives of the functions $a^{i j}$.

Notice that this lemma is also true if $U$ is only twice differentiable in some domain. (For any point in the domain choose $\phi \in C_{0}^{\infty}$ (domain) such that $\phi \equiv 1$ in a neighborhood of the point, then consider $\phi U$ and extend $\phi U$ to all of $\mathbf{R}^{m}$. The derivatives of $\phi U$ at the point in question equal the derivatives of $U$ and $M$ does not depend on $U$.)

We consider equations of the form

$$
L[u]=a^{i j}(x, t) U_{, i j}+b^{i}(x, t) U_{, i}+C U+U_{, t}=0
$$

on a domain $\Omega=D \times T$ where $D$ is a bounded domain in $\mathbf{R}^{n}$ and $T=\left\{t_{1}<\right.$ $\left.t<t_{2}\right)$ is an open interval. We employ the auxiliary function $W=\left(U_{, k} U_{, k}+\right.$ $\left.\left(U_{t}\right)^{2}+1\right) e^{N t}$ where $N$ is a positive constant to be chosen. We prove

THEOREM 5. Suppose that

(1) $\left(a^{i j}\right)$ is a positive semidefinite symmetric matrix and $\left(a^{i j}(x, t)\right)$ may be extended as a positive semidefinite matrix to $\mathbf{R}^{n} \times \mathbf{R}$ such that $a^{i j} \in C^{2}\left(\mathbf{R}^{n} \times\right.$ $\mathbf{R})$, with bounded second order derivatives.

(2) $b^{i}$ and $C$ are in $C^{1}(\Omega)$ and are bounded along with their first partial derivatives. 
(3) $U \in C^{3}(\Omega)$ and $U$ uniformly bounded in $\Omega$.

Then if $U$ satisfies equation (3.1) the constant $N$ may be chosen sufficiently large so that $L[W] \geqslant 0$.

Proof. The proof consists of calculating $L[W]$ and then obtaining $L[W] \geqslant$ 0 through a series of inequalities.

$$
\begin{aligned}
e^{-N t} L[W]= & 2 a^{i j} U_{, k i} U_{, k j}+2 a^{i j} U_{, t i} U_{, t j}+2 a^{i j} U_{, k} U_{, k i j} \\
& +2 a^{i j} U_{, t} U_{, t i j}+2 b^{i} U_{, k} U_{, k i}+2 b^{i} U_{, t} U_{, t i}+2 U_{, k} U_{, k t} \\
& +2 U_{, t} U_{, t t}+N|\operatorname{grad} U|^{2}+N+C|\operatorname{grad} U|^{2}+C .
\end{aligned}
$$

From the equation $L[U]=0$ we have by differentiation, multiplication by $2 U_{, k}$ and summing on $k$,

$$
\begin{aligned}
& 2 a^{i j} U_{, i j k} U_{, k}+2 a_{, k}^{i j} U_{, i j} U_{, k}+2 b^{i} U_{, i k} U_{, k} \\
&+2 b_{, k}^{i} U_{, i} U_{, k}+2 U_{, k} U_{, k}+2 C U_{, k} U_{, k}+2 C_{, k} U U_{, k}=0
\end{aligned}
$$

Similarly, we arrive at

$$
\begin{aligned}
2 a^{i j} U_{, i j t} U_{, t}+2 a_{, t}^{i j} U_{, i j} U_{, t}+2 b^{i} U_{, i t} U_{, t} \\
+2 b_{, t}^{i} U_{, i} U_{, t}+2 U_{, t} U_{, t}+2 C U_{, t} U_{, t}+2 C_{, t} U U_{, t}=0
\end{aligned}
$$

Using equations (3.2) and (3.3) we obtain

$$
\begin{aligned}
e^{-N t} L[W]= & 2 a^{i j} U_{, k j} U_{, k i}+2 a^{i j} U_{, t j} U_{, t i}-2 a_{, k}^{i j} U_{, i j} U_{, k} \\
& -2 a_{, t}^{i j} U_{, i j} U_{, t}-2 b_{, k}^{i} U_{, i} U_{, k}-2 b_{, i}^{i} U_{, i} U_{, t}-C|\operatorname{grad} U|^{2} \\
& -2 C_{, k} U U_{, k}-2 C_{, t} U U_{, t}+C+N+N|\operatorname{grad} U|^{2} .
\end{aligned}
$$

We can bound terms from the above expression as follows:

$$
\left|2 b_{, k}^{i} U_{, i} U_{, k}+2 b_{, t}^{i} U_{, i} U_{, t}\right| \leqslant \kappa|\operatorname{grad} U|^{2}
$$

where $\kappa$ is a constant depending on the bounds for the coefficients and their derivatives and the bound on $U$. We also have

$$
\left|2 a_{, k}^{i j} U_{, i j} U_{, k}\right| \leqslant \sum_{k} \varepsilon\left(a_{, k}^{i j} U_{, i j}\right)^{2}+\varepsilon^{-1}|\operatorname{grad} U|^{2}
$$

where $\varepsilon$ is any positive number. Similarly

$$
\left|2 a_{, i}^{i j} U_{, i j} U_{, t}\right| \leqslant \varepsilon\left(a_{, i}^{i j} U_{, i j}\right)^{2}+\varepsilon^{-1}|\operatorname{grad} U|^{2} .
$$

We apply the lemma stated before the theorem to the first inequality above and, by choosing $\varepsilon=1 / n M$, arrive at 


$$
\left|2 a_{, k}^{i j} U_{, i j} U_{, k}\right| \leqslant a^{i j} U_{, k i} U_{, k j}+n M|\operatorname{grad} U|^{2} .
$$

To apply the lemma to the second inequality (i.e. to $\left.\left(a_{, i}^{i j} U_{, i j}\right)^{2}\right)$ we extend $a^{i j}$ to an $(n+1) \times(n+1)$ matrix by adding a column and a row of zeros. Applying the lemma we obtain

$$
\left(a_{, i}^{i j} U_{, i j}\right)^{2} \leqslant M a^{i j} U_{, k i} U_{, k j}+M a^{i j} U_{, i i} U_{, j}
$$

and therefore

$$
\left|2 a_{, i}^{i j} U_{, i j} U_{, t}\right| \leqslant a^{i j} U_{, k i} U_{, k j}+a^{i j} U_{, i i} U_{, j}+M|\operatorname{grad} U|^{2}
$$

(choosing $\varepsilon=1 / M$ ).

Now using all the inequalities indicated by asterisks we arrive at

$$
\begin{aligned}
e^{-N t} L[W] \geqslant & a^{i j} U_{, t i} U_{, t j}-n M|\operatorname{grad} U|^{2}-M|\operatorname{grad} U|^{2} \\
& -3 \kappa|\operatorname{grad} U|^{2}-2 \kappa+N+N|\operatorname{grad} U|^{2} .
\end{aligned}
$$

It is clear that by choosing $N$ sufficiently large (e.g., $N=n M+M+3 \kappa$ ), $e^{-N t} L[W] \geqslant 0$ and therefore $L[W] \geqslant 0$.

REMARK. Once we know $L[W] \geqslant 0$ then, depending on what additional conditions $L$ satisfies, we may apply either Theorem 3 or Theorem 4 to obtain maximum principles for $W$. Also note that Theorems 3 and 4 may be used to obtain the necessary uniform bound on $U$ (necessary for Theorem 5 ) in terms of boundary values for $U$.

We can extend the last theorem in two ways. First we state a theorem for higher order derivatives whose proof is analogous to the above theorem. Second we prove a theorem on the gradient for certain systems. For the theorem on higher order derivatives we consider the auxiliary function $W=\Sigma_{1<|\alpha| \leqslant K}\left[U_{, \alpha} U_{, \alpha}+1\right] e^{N t}$ where $N$ is a positive constant to be chosen and $\alpha$ is an $n+1$ dimensional multi-index (i.e. may include derivatives with respect to $t$ ).

\section{THEOREM 6. Suppose that}

(1) $\left(a^{i j}(x, t)\right)$ is a positive semidefinite symmetric matrix, that $\left(a^{i j}\right)$ may be extended as a positive semidefinite matrix to $\mathbf{R}^{n} \times \mathbf{R}$ in such a way that $a^{i j}(x, t)$ has bounded second order derivatives in $\mathbf{R}^{n} \times \mathbf{R}$, that $a^{i j}(x, t) \in$ $C^{K}(\Omega)$ and all derivatives up to order $K$ are bounded, and that

(2) $b^{i}(x, t)$ and $C(x, t)$ are in $C^{K}(\Omega)$ and are bounded along with their partial derivatives up to order $K$. Also, suppose that

(3) $U \in C^{K}(\Omega)$ and $U$ is uniformly bounded in $\Omega$.

Then if $U$ satisfies (3.1) the constant $N$ may be chosen sufficiently large so that $L[W] \geqslant 0$.

Proof. The proof is very similar to the previous proof for the gradient. We first calculate $L[W]$. In what follows repeated multi-indices $\alpha$ are summed 
over $1 \leqslant|\alpha| \leqslant K$. We have

$$
\begin{aligned}
e^{-N t} L[W]= & 2 a^{i j} U_{, \alpha i j} U_{, \alpha}+2 a^{i j} U_{, \alpha i} U_{, \alpha j}+2 b^{i} U_{, \alpha i} U_{, \alpha} \\
& +C U_{, \alpha} U_{, \alpha}+C+2 U_{, \alpha t} U_{, \alpha}+N U_{, \alpha} U_{, \alpha}+N .
\end{aligned}
$$

From the equation $L[U]=0$ we obtain

$$
\begin{gathered}
2 \sum_{1<|\alpha|<K} \sum_{p+q=\alpha} \frac{\alpha !}{p ! q !}\left(a_{p}^{i j} U_{, i j q} U_{, \alpha}+b_{p}^{i} U_{, i q} U_{, \alpha}+C_{p} U_{, q} U_{, \alpha}\right) \\
+2 U_{, t} U_{, \alpha}=0 .
\end{gathered}
$$

Subtracting this equation from the above equation for $e^{-N t} L[W]$ we obtain

$$
\begin{aligned}
& e^{-N t} L[W]=2 a^{i j} U_{, \alpha i} U_{, \alpha j}+C+N U_{, \alpha} U_{, \alpha}+N \\
& -2 \sum_{1<|\alpha|<K} \sum_{\substack{p+q=\alpha \\
|p|>2}}\left[\frac{\alpha !}{p ! q !}\left(a_{, p}^{i j} U_{, i j q} U_{, \alpha}+b_{p}^{i} U_{, i q} U_{, \alpha}\right)+C_{p} U_{, q} U_{, \alpha}\right] \\
& -2 \sum_{1<|\alpha|<K} \sum_{\substack{p+q=\alpha \\
|p|=1}} \frac{\alpha !}{p ! q !}\left(b_{p}^{i} U_{, i q} U_{, \alpha}+C_{p} U_{, q} U_{, \alpha}\right) \\
& -2 \sum_{1<|\alpha|<K} \sum_{\substack{p+q=\alpha \\
|p|=1}} \frac{\alpha !}{p ! q !}\left(a_{, p}^{i j} U_{, i j q} U_{, \alpha}\right) .
\end{aligned}
$$

The coefficient $C$ may be bounded by $N$. The first two indicated double sums may be bounded by $N U_{, \alpha} U_{, \alpha}+N$ since the coefficients and $U$ are uniformly bounded. Since in the last double sum, $|p|=1$, we may employ the Oleinik and Radkevič lemma as in the previous theorem. Thus for $N$ large enough $L[W] \geqslant 0$.

As before we note that Theorems 3 and 4 may be applied to $W$ provided $L$ satisfies the appropriate conditions.

In the final two theorems we consider weakly-coupled systems of equations of the form

$$
\begin{array}{r}
L^{\mu}(\bar{U})=a^{i j}(x, t) U_{, i j}^{\mu}+b^{i}(x, t) U_{, i}^{\mu}+U_{, t}^{\mu}+C^{\mu \nu}(x, t) U^{\nu}=0, \\
\mu=1, \ldots, m,
\end{array}
$$

in a domain $D=\Omega \times T$.

Repeated Latin sub and superscripts are summed from 1 to $n$ and repeated Greek superscripts are summed from 1 to $m$.

THEOREM 7. Assume that $\left(a^{i j}\right)$ is a positive semidefinite matrix and that $C^{\mu \nu}(x, t)$ is uniformly bounded in $D$ for all $\mu$ and $\nu$. Let $v=e^{N t} U^{\mu} U^{\mu}$. If $\bar{U}$ satisfies system (3.4) then $L[v]=a^{i j} v_{, i j}+b^{i} v_{i}+v_{, t} \geqslant 0$ provided that $N$ is chosen sufficiently large. If $C^{\mu \nu}$ is negative semidefinite then $N$ may be chosen to be zero. 
Proof. We have

$$
\begin{aligned}
e^{-N t}\left(a^{i j} v_{, i j}+b^{i} v_{, i}+v_{, t}\right)= & 2 a^{i j} U^{\mu} U_{, i j}^{\mu}+2 a^{i j} U_{, i} U_{j} \\
& +2 b^{i} U^{\mu} U_{, i}^{\mu}+2 U^{\mu} U_{, t}^{\mu}+N U^{\mu} U^{\mu} .
\end{aligned}
$$

Multiplying $L[U]=0$ by $2 U^{\mu}$ and summing over $\mu$, we get

$$
2 a^{i j} U_{, i j}^{\mu} U^{\mu}+2 b^{i} U_{, i}^{\mu} U^{\mu}+2 U_{, t}^{\mu} U^{\mu}+2 C^{\mu \nu} U^{\nu} U^{\mu}=0 \text {. }
$$

Using this equation we obtain

$$
e^{-N t} L[v]=2 a^{i j} U_{, i} U_{j}+N U^{\mu} U^{\mu}-C^{\mu \nu} U^{\mu} U^{\nu} .
$$

Clearly the right-hand side is nonnegative for $N$ large enough and $N$ may be taken to be zero if $\left(C^{\mu \nu}\right)$ is negative semidefinite. Thus

$$
L[v]=a^{i j} v_{, i j}+b^{i} v_{, i}+v_{, t} \geqslant 0 .
$$

REMARK. If $L$ satisfies the conditions of either Theorem 3 or Theorem 4, then we know that $v=e^{N t} U^{\mu} U^{\mu}$ will satisfy the maximum principles of Bony.

In the last theorem we consider the auxiliary function $W=e^{N t}\left(U_{, k}^{\mu} U_{, k}^{\mu}+\right.$ $\left.U_{, t}^{\mu} U_{, t}^{\mu}+1\right)$.

THEOREM 8. Suppose that

(1) $\left(a^{i j}\right)$ is a positive semidefinite symmetric matrix, that $\left(a^{i j}(x, t)\right)$ may be extended as a positive semidefinite matrix to $\mathbf{R}^{n} \times \mathbf{R}$ in such a way that $a^{i j}$ is an element of $C^{2}\left(\mathbf{R}^{n} \times \mathbf{R}\right)$ with bounded second derivatives, and that

(2) $b^{i}$ and $C^{\mu \nu}$ are in $C^{1}(\Omega)$ and are bounded along with their first partial derivatives. Also, suppose that

(3) $U^{\mu} \in C^{3}(\Omega)$ for $1 \leqslant \mu \leqslant m$ and $U^{\mu} U^{\mu}$ is uniformly bounded in $\Omega$. Then if $\bar{U}$ satisfies system (3.4) $N$ may be chosen sufficiently large so that

$$
L[W]=a^{i j} W_{, i j}+b^{i} W_{, i}+W_{, t} \geqslant 0 .
$$

Proof. We have

$$
\begin{aligned}
e^{-N t} L[W]= & e^{-N t}\left(a^{i j} W_{, i j}+b^{i} W_{, i}+W_{, t}\right)=2 a^{i j} U_{, i k}^{\mu} U_{, j k}^{\mu}+2 a^{i j} U_{, t i}^{\mu} U_{, t j}^{\mu} \\
& +2 a^{i j} U_{, k i j}^{\mu} U_{i k}^{\mu}+2 a^{i j} U_{, t i j}^{\mu} U_{, t}^{\mu}+2 b^{i} U_{, k i}^{\mu} U_{, k}^{\mu}+2 b^{i} U_{, t i}^{\mu} U_{, t}^{\mu} \\
& +2 U_{, k t}^{\mu} U_{, k}^{\mu}+2 U_{, t t}^{\mu} U_{, t}^{\mu}+N U_{, k}^{\mu} U_{, k}^{\mu}+N U_{, t}^{\mu} U_{, t}^{\mu}+N .
\end{aligned}
$$

We next make use of the equation $L^{\mu}[U]=0$. As in the scalar case we differentiate this equation with respect to $x_{k}$, multiply by $U_{, k}^{\mu}$ and then sum with repeat to $k$. Similarly we differentiate $L^{\mu}[U]=0$ with respect to $t$ and multiply by $U_{, t}^{\mu}$ and sum. Using the resulting two equations we obtain

$$
\begin{aligned}
e^{-N t} L[W]= & 2 a^{i j} U_{, i k}^{\mu} U_{j k}^{\mu}+2 a^{i j} U_{, i t}^{\mu} U_{, j}^{\mu}+N U_{, k}^{\mu} U_{, k}^{\mu} \\
& +N U_{, t}^{\mu} U_{, t}^{\mu} N-2 C^{\mu \nu} U_{, k}^{\nu} U_{, k}^{\mu}-2 C^{\mu \nu} U_{, t}^{\nu} U_{, t}^{\mu} \\
& -2 C_{, k}^{\mu \nu} U^{\nu} U_{, k}^{\mu}-2 C_{, t}^{\mu \nu} U^{\nu} U_{, t}^{\mu}-2 a_{, k}^{i} U_{, i j}^{\mu} U_{, k}^{\mu}-2 a_{, t}^{i} U_{, i j}^{\mu} U_{, t}^{\mu}
\end{aligned}
$$


Using estimates exactly like those in the scalar case it is easily seen that for $N$ large enough, $L[W] \geqslant 0$.

\section{BIBLIOGRAPHY}

1. A. Friedman, Partial differential equations, Holt, Rinehart and Winston, New York, 1969.

2. C. Miranda, Sul teorema del massimo modulo per una classe di sistemi ellittici di equazioni del secondo ordine e per le equazioni a coefficienti complessi, Ist. Lombardo Accad. Sci. Lett. Rend. A 104 (1970), 736-745. MR 45 \#5557.

3. G. Stampacchia, Le probleme de Dirichlet pour les équations elliptiques du second ordre $\grave{a}$ coefficients discontinus, Ann. Inst. Fourier (Grenoble) 15 (1965), fasc. 1, 189-258. MR 33 \#404.

4. A. M. I''in, A. S. Kalašnikov and O. A. Oleǐnik, Second-order linear equations of parabolic type, Uspehi Mat. Nauk 17 (1962), no. 3 (105), 3-145 = Russian Math. Surveys 17 (1962), no. 3, 1-143. MR 25 \#2328.

5. O. A. Oleinik and E. V. Radkevič, Equations of second order with nonnegative characteristic form, Itogi Nauki. Mat. Anal. 1969, VINITI, Moscow, 1971; English transl., Plenum Press, New York, 1973.

6. M. H. Protter and H. F. Weinberger, Maximum principles in differential equations, PrenticeHall, Englewood Cliffs, N.J., 1967. MR 36 \#2935.

7. __ A maximum principle and gradient bounds for linear elliptic equations, Indiana Univ. Math. J. 23 (1973/74), 239-249. MR 48 \#2556.

8. J.-M. Bony, Principe du maximum, inégalité de Harnack et unicité du problème de Cauchy pour les opérateurs elliptiques dégénérés, Ann. Inst. Fourier (Grenoble) 19 (1969), fasc. 1, 277-304. MR 41 \# 7486.

9. A. D. Aleksandrov, Investigations on the maximum principle. I, II, III, IV, V, Izv. Vysš. Učbn. Zaved. Matematika 1958, no. 5 (6), 126-157; ibid. 1959, no. 3 (10), 3-12; ibid. 1959, no. 5 (12), 16-32; ibid. 1960, no. 3 (16), 3-15; ibid. 1960, no. 5 (18), 16-26. MR 24 \#A3400a, b, c, d, e.

Department of Mathematics, Texas A\&M University, College Station, TeXas 77843 